\title{
RELATIVISTIC SUPPRESSION OF BLACK HOLE RECOILS
}

\author{
Michael Kesden ${ }^{1}$, Ulrich Sperhake ${ }^{1,2}$, and Emanuele Berti ${ }^{1,2}$ \\ ${ }^{1}$ California Institute of Technology, MC 350-17, 1216 E. California Blvd., Pasadena, CA 91125, USA \\ ${ }^{2}$ Department of Physics and Astronomy, The University of Mississippi, University, MS 38677-1848, USA \\ Received 2010 March 24; accepted 2010 April 14; published 2010 May 5
}

\begin{abstract}
Numerical-relativity simulations indicate that the black hole produced in a binary merger can recoil with a velocity up to $v_{\max } \simeq 4000 \mathrm{~km} \mathrm{~s}^{-1}$ with respect to the center of mass of the initial binary. This challenges the paradigm that most galaxies form through hierarchical mergers, yet retain supermassive black holes (SBHs) at their centers despite having escape velocities much less than $v_{\max }$. Interaction with a circumbinary disk can align the binary black hole spins with their orbital angular momentum, reducing the recoil velocity of the final black hole produced in the subsequent merger. However, the effectiveness of this alignment depends on highly uncertain accretion flows near the binary black holes. In this paper, we show that if the spin $\mathbf{S}_{1}$ of the more massive binary black hole is even partially aligned with the orbital angular momentum $\mathbf{L}$, relativistic spin precession on sub-parsec scales can align the binary black hole spins with each other. This alignment significantly reduces the recoil velocity even in the absence of gas. For example, if the angle between $\mathbf{S}_{1}$ and $\mathbf{L}$ at large separations is $10^{\circ}$ while the second spin $\mathbf{S}_{2}$ is isotropically distributed, the spin alignment discussed in this paper reduces the median recoil from $864 \mathrm{~km} \mathrm{~s}^{-1}$ to $273 \mathrm{~km} \mathrm{~s}^{-1}$ for maximally spinning black holes with a mass ratio of $9 / 11$. This reduction will greatly increase the fraction of galaxies retaining their SBHs.
\end{abstract}

Key words: black hole physics - galaxies: evolution - gravitational waves

Online-only material: color figures

\section{INTRODUCTION}

Observations suggest that most galaxies host supermassive black holes (SBHs) at their centers whose masses are tightly correlated with properties of their host spheroids (Magorrian et al. 1998; Ferrarese \& Merritt 2000; Tremaine et al. 2002). If galaxies form through hierarchical mergers, their SBHs may form from the merger of the smaller SBHs in their progenitor galaxies. The final stage of these mergers involves highly curved, dynamical spacetimes that can only be simulated in numerical relativity (NR). Following a major breakthrough in 2005 (Pretorius 2005; Campanelli et al. 2006; Baker et al. 2005), numerical relativists can now accurately determine the anisotropic emission of gravitational waves during the final stage of black hole mergers. When gravitational waves are preferentially emitted in one direction, the conservation of linear momentum requires that the final black hole produced in the merger recoil in the opposite direction. These recoil velocities or "kicks" can approach $4000 \mathrm{~km} \mathrm{~s}^{-1}$ for maximally spinning mergers (Campanelli et al. 2007a; Gonzalez et al. 2007a). Kicks this large exceed the escape velocities of even the most massive galaxies and would thus eject SBHs from their hosts (Merritt et al. 2004; Schnittman \& Buonanno 2007; Schnittman 2007). Frequent SBH ejections would seem to contradict the tightness of the observed correlations between SBHs and their host galaxies. Kicks would pose an even greater problem at high redshifts, where typical galactic escape velocities decrease while recoils remain a fixed fraction of the speed of light.

How might we avoid black hole mergers that lead to large kicks? To answer this question, we must take a closer look at how the predicted recoils depend on the dimensionless spins $\chi_{i} \equiv \mathbf{S}_{i} / m_{i}^{2}$ and mass ratio $q \equiv m_{2} / m_{1} \leqslant 1$ of the merging black holes. Reliable NR simulations have been performed for $q \geqslant 0.1$ and $\left|\chi_{i}\right| \leqslant 0.9$; in this range, the recoils are well described by the fitting formula (Campanelli et al. 2007a)

$$
\vec{v}\left(q, \chi_{1}, \chi_{2}\right)=v_{m} \hat{\mathbf{e}}_{1}+v_{\perp}\left(\cos \xi \hat{\mathbf{e}}_{1}+\sin \xi \hat{\mathbf{e}}_{2}\right)+v_{\|} \hat{\mathbf{e}}_{z},
$$

where $v_{m}=A \eta^{2} \frac{1-q}{1+q}(1+B \eta), v_{\perp}=H \eta^{2} \boldsymbol{\Delta}^{\|} \cdot \hat{\mathbf{e}}_{z}$ and $v_{\|}=$ $K \eta^{2} \cos \left(\Theta-\Theta_{0}\right)\left|\Delta^{\perp}\right|$. Here $\left(\hat{\mathbf{e}}_{1}, \hat{\mathbf{e}}_{2}, \hat{\mathbf{e}}_{z}\right)$ are an orthonormal basis with $\hat{\mathbf{e}}_{z}$ parallel to the orbital angular momentum $\mathbf{L}$, $\eta \equiv q /(1+q)^{2} \leqslant 1 / 4$ is the symmetric mass ratio, and $\Delta^{\|, \perp}$ are the components of

$$
\Delta \equiv \frac{q \chi_{2}-\chi_{1}}{1+q}
$$

parallel and perpendicular to $\mathbf{L}$. $\Theta$ is the angle between $\Delta^{\perp}$ and the separation $\mathbf{r}$ of the two black holes "at merger." NR simulations indicate that the best-fit values for the coefficients appearing in the above formula are $A=1.2 \times 10^{4} \mathrm{~km} \mathrm{~s}^{-1}, B=$ -0.93 (Gonzalez et al. 2007b), $H=(6.9 \pm 0.5) \times 10^{3} \mathrm{~km} \mathrm{~s}^{-1}$ (Lousto \& Zlochower 2008), and $K=(6.0 \pm 0.1) \times 10^{4} \mathrm{~km} \mathrm{~s}^{-1}$ (Campanelli et al. 2007b). The angle $\xi \sim 145^{\circ}$ for a wide range of quasi-circular configurations (Lousto \& Zlochower 2008), while $\Theta_{0}$ depends on the mass ratio $q$ but not on the spins (Lousto $\&$ Zlochower 2009). The large value of $K$ implies that equalmass mergers with maximal spins pointed in opposite directions in the orbital plane generate a recoil of $K / 16=3750 \mathrm{~km} \mathrm{~s}^{-1}$.

The most obvious way to avoid these large kicks is to require black holes to be non-spinning:

$$
\chi_{i}=0 \rightarrow \Delta=0 \rightarrow v_{\perp}=v_{\|}=0
$$

In this case, $v_{m}$ is maximized at the modest value $175 \pm$ $11 \mathrm{~km} \mathrm{~s}^{-1}$ for a mass ratio $q \simeq 0.36$ (Gonzalez et al. 2007b). However, theory shows that non-spinning black holes can be spun up to the Kerr limit $|\chi|=1$ by steady accretion after 
increasing their mass by only a factor of $\sqrt{6}$ (Bardeen 1970). Observations of $\mathrm{Fe} \mathrm{K} \alpha$ fluorescence can be used to measure black hole spins (Reynolds et al. 1999) and indicate that real SBHs can approach this limit: for the SBH in the Seyfert galaxy MCG-06-30-15 the measured spin is $|\chi|=0.989_{-0.002}^{+0.009}$ at $90 \%$ confidence (Brenneman \& Reynolds 2006; Berti et al. 2009).

If black holes are highly spinning, the recoil can be reduced by aligning the spins and thus $\Delta$ with $\mathbf{L}$ :

$$
\chi_{i} \| \mathbf{L} \rightarrow \Delta^{\perp}=0 \rightarrow v_{\|}=0 .
$$

This spin configuration leads to smaller kicks, since the coefficient $H$ is almost an order of magnitude less than $K$. The merger of equal-mass, maximally spinning black holes with one spin aligned with $\mathbf{L}$ and the other anti-aligned generates a recoil of $H / 16=431 \mathrm{~km} \mathrm{~s}^{-1}$. Gaseous accretion disks are needed to provide dynamical friction to allow SBHs separated by $r \simeq 1$ pc to merge in less than a Hubble time (Begelman et al. 1980). These same accretion disks can exert torques on the SBHs which align their spins and orbital angular momentum with that of the disk, thus producing the desired aligned spin configuration (Bogdanović et al. 2007). However, the effectiveness of this alignment mechanism depends on the highly uncertain nature of the accretion flow near the merging black holes. Dotti et al. (2010) find a residual misalignment of $10^{\circ}\left(30^{\circ}\right)$ between the black hole spins and accretion disk depending on whether the disk is cold (hot). This misalignment could be even greater in a gas-poor merger or one in which accretion onto the SBHs proceeds through a series of small-scale, randomly oriented events (King \& Pringle 2007; Berti \& Volonteri 2008).

In this paper, we present a new mechanism to reduce gravitational recoils by aligning black hole spins with each other prior to merger. Boyle et al. (2008) and Boyle \& Kesden (2008) showed that the symmetries of binary black hole systems imply that recoils are only generated by a weighted difference of the two spins. This general result can be seen to hold for the fitting formula of Campanelli et al. (2007a) by noting that a weighted difference of spins appears in the numerator of $\Delta$ in Equation (2). Spin alignment is a consequence of relativistic spin precession as the black holes inspiral due to the loss of energy and angular momentum to gravitational radiation (GR). We begin calculating the inspiral at an initial separation $r_{i}=500 R_{S}$ where spin alignment begins for comparablemass binaries (Schnittman 2004) and end at a final separation $r_{f}=5 R_{S}$ near where NR simulations typically begin. Here $R_{S}=2 G M / c^{2}$ is the Schwarzschild radius of a non-spinning black hole of mass $M$. Relativists use units in which $G=c=1$, allowing them to measure distance and time in units of $M$, where $M \equiv m_{1}+m_{2}$ is the sum of the masses of the merging black holes. We shall do this for the rest of the paper. The spin alignment discussed in this paper occurs for both gas-rich and gas-poor mergers, as GR dominates the dynamics even in the presence of gas at binary separations less than

$$
r_{\mathrm{GR}} \sim 3000 M q^{1 / 4}\left(\frac{\dot{M}}{1 M_{\odot} \mathrm{yr}^{-1}}\right)^{-1 / 4},
$$

where $\dot{M}$ is the rate of gas infall (Begelman et al. 1980).

We briefly describe the relativistic dynamics leading to spin alignment in Section 2; readers interested in further details can find them in our longer paper on how spin alignment affects the distributions of black hole final spins (Kesden et al. 2010). The most notable effect of spin alignment is to suppress the recoil velocity when the spin of the larger black hole is initially partially aligned with $\mathbf{L}$. The magnitude of this suppression for distributions with different mass ratios and initial spins is presented in Section 3. Some concluding remarks are provided in Section 4.

\section{SPIN ALIGNMENT}

To understand why black hole spins align, we must first describe how they precess at separations $r<r_{\mathrm{GR}}$, where the inspiral is determined predominantly by the loss of energy and angular momentum to GR. When the orbital speed is much less than the speed of light, the motion of the black holes, the precession of their spins, and the emission of GR can all be calculated in the post-Newtonian (PN) limit. We use the PN equations for precessing binaries first derived in Kidder (1995), supplemented with the quadrupole-monopole interaction considered in Racine (2008). GR circularizes eccentric orbits (Peters \& Mathews 1963), so we restrict our attention to quasi-circular orbits of slowly decreasing radius. In the PN limit $r \gg M$, the orbital period $t_{\text {orb }} \propto r^{3 / 2}$ (Kepler's third law), the spin precession period $t_{p} \sim\left|\Omega_{i}\right|^{-1} \propto r^{5 / 2}$, and the radiation reaction time $t_{r} \sim r / \dot{r} \propto r^{4}$. These scalings imply that $t_{\text {orb }} \ll t_{p} \ll t_{r}$, allowing several simplifications. Since $t_{\text {orb }} \ll t_{p}$, the spins $\mathbf{S}_{i}$ precess according to

$$
\frac{d \mathbf{S}_{i}}{d t}=\boldsymbol{\Omega}_{i} \times \mathbf{S}_{i},
$$

where $\Omega_{i}$ are the orbit-averaged spin precession frequencies. These depend on the mass ratio $q$, the orbital angular momentum $\mathbf{L}$, and the spins $\mathbf{S}_{1}$ and $\mathbf{S}_{2}$ as shown in Equation (2.2) of Kesden et al. (2010). Since $t_{p} \ll t_{r}$, the total angular momentum $\mathbf{J}=\mathbf{L}+\mathbf{S}_{1}+\mathbf{S}_{2}$ and magnitude $|\mathbf{L}|$ are constant on the timescale $t_{p}$. This implies that the direction of $\mathbf{L}$ evolves as

$$
\frac{d \hat{\mathbf{L}}}{d t}=-\frac{1}{|\mathbf{L}|}\left(\frac{d \mathbf{S}_{1}}{d t}+\frac{d \mathbf{S}_{2}}{d t}\right) .
$$

The magnitude $|\mathbf{L}|$ does decrease on the longer timescale $t_{r}$ as the orbital frequency $\omega$ increases according to Equation (2.6) of Kesden et al. (2010).

Schnittman (2004) discovered that if black holes inspiral as described above, spin precession and radiation reaction align $\mathbf{S}_{1}$ and $\mathbf{S}_{2}$ with each other if $\theta_{1}<\theta_{2}$, where $\theta_{1}\left(\theta_{2}\right)$ is the angle between $\mathbf{L}$ and $\mathbf{S}_{1}\left(\mathbf{S}_{2}\right)$. Conversely, if $\theta_{1}>\theta_{2}, \mathbf{S}_{1}$ and $\mathbf{S}_{2}$ will become anti-aligned with each other. This alignment is strongest for mass ratios $q$ near unity, though it vanishes for precisely equal masses as there is no distinction between $\mathbf{S}_{1}$ and $\mathbf{S}_{2}$ in that case. We show the magnitude of this alignment for maximally spinning black holes $\left(\left|\chi_{i}\right|=1\right)$ in Figure 1 and for black holes with $\left|\chi_{i}\right|=0.5$ in Figure 2. The upper panels show black holes with the nearly equal mass ratio $q=9 / 11$, while the lower panels refer to $q=1 / 3$. The black curves show that black holes with isotropic spin distributions at $r_{i}=1000 M$ (flat distributions in $\cos \theta_{1}, \cos \theta_{2}, \Delta \phi \equiv \phi_{2}-\phi_{1}$, and $\cos \theta_{12}$, the angle between $\mathbf{S}_{1}$ and $\mathbf{S}_{2}$ ) maintain these isotropic distributions as they inspiral to $r_{f}=10 \mathrm{M}$, consistent with previous studies of precessing spin distributions (Bogdanović et al. 2007; Herrmann et al. 2010; Lousto et al. 2009). Isotropic spin distributions will have $\theta_{1}<\theta_{2}$ just as often as $\theta_{1}>\theta_{2}$, implying that just as many spins will become aligned as anti-aligned during the inspiral.

These isotropic spin distributions at $r_{i}=1000 M$ are only expected for the most gas-poor mergers; in the presence of gas, accretion torques will partially align the spins and orbital 

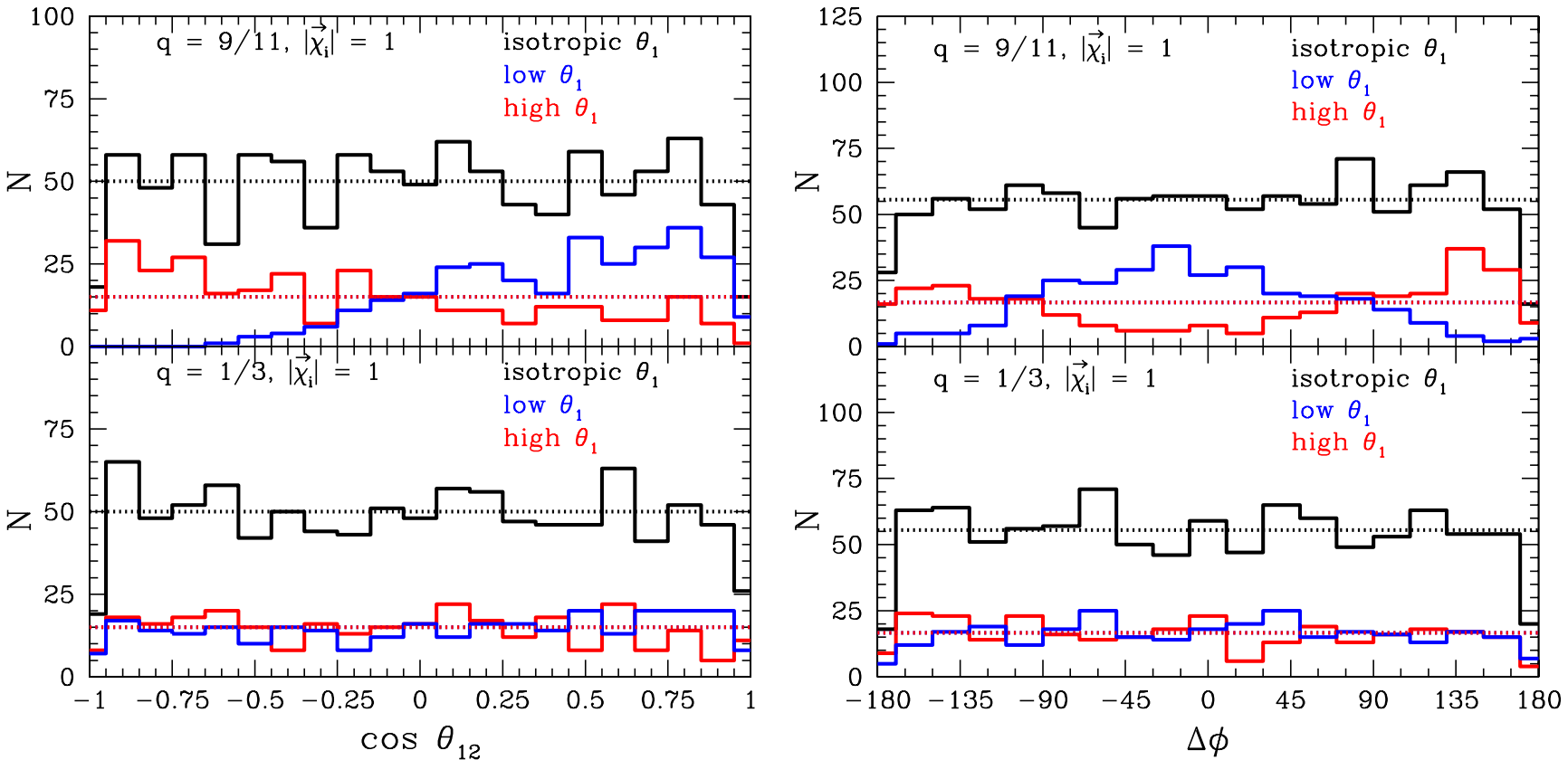

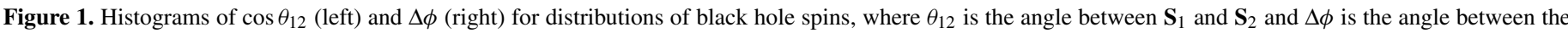

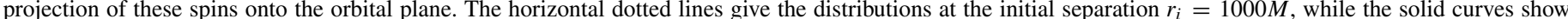

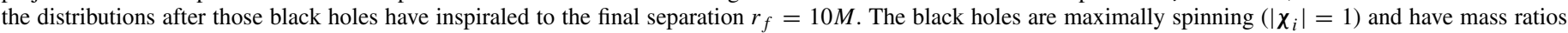

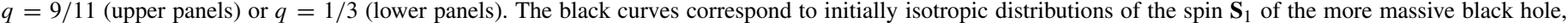
while the blue (red) curves show subsets of this distribution with the $30 \%$ lowest (highest) initial values of $\theta_{1}$, the angle between $\mathbf{S}_{1}$ and $\mathbf{L}$.

(A color version of this figure is available in the online journal.)
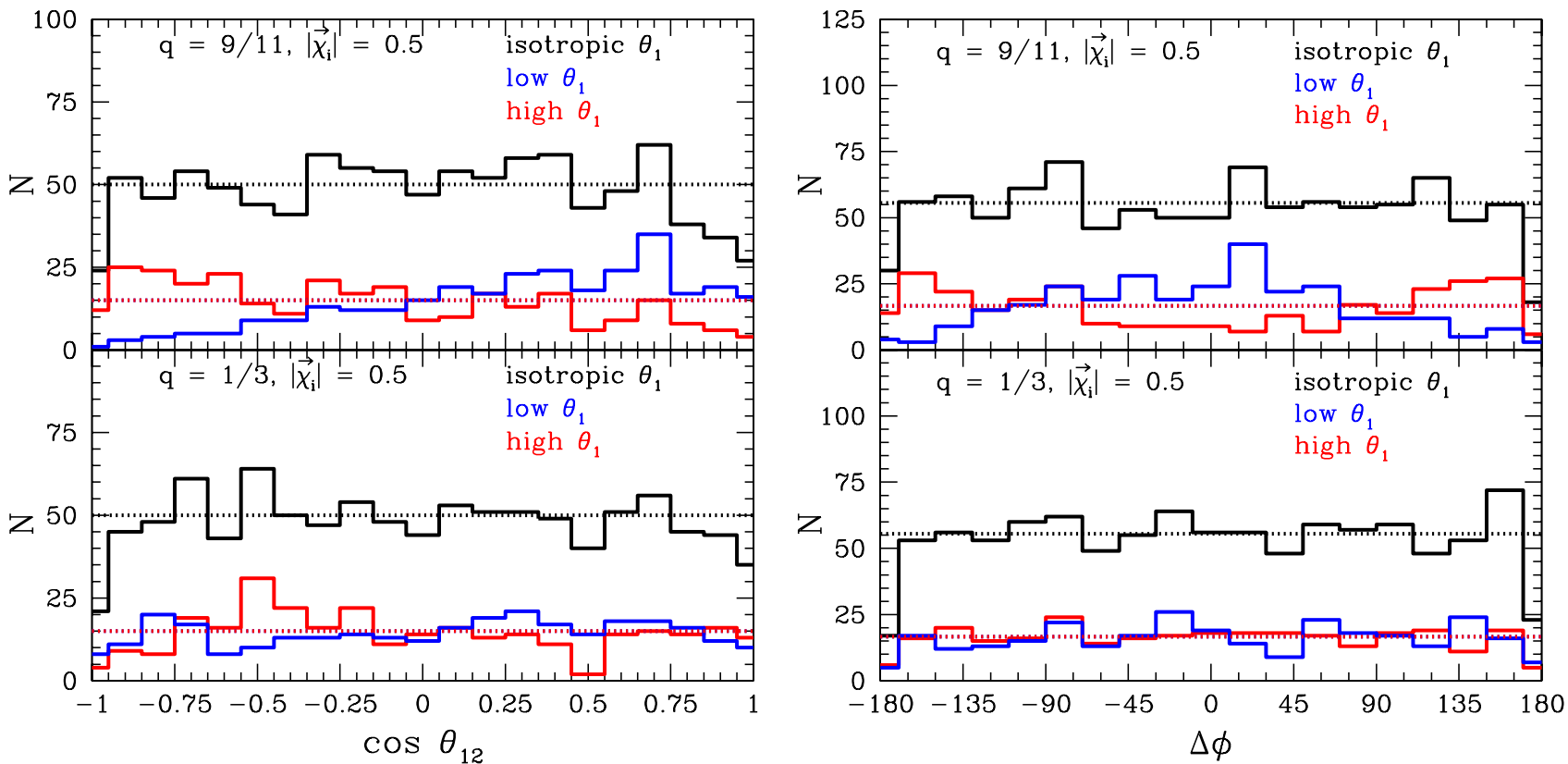

Figure 2. Histograms of the same quantities shown in Figure 1, except the black holes now have initial spin magnitudes $\left|\chi_{i}\right|=0.5$.

(A color version of this figure is available in the online journal.)

angular momentum with that of the disk (Bogdanović et al. 2007). We consider a scenario in which the spin $\mathbf{S}_{1}$ of the more massive black hole is partially aligned with $\mathbf{L}$ while the other spin $\mathbf{S}_{2}$ remains isotropically distributed. This scenario is consistent with that explored by Chang et al. (2009) and Dotti et al. (2010), where the more massive black hole or "primary" is at rest in the center of the accretion disk while the "secondary" migrates inward. The blue (red) curves in Figures 1 and 2 show the subset of black hole binaries with the $30 \%$ lowest (highest) values of $\theta_{1}$ at $r_{i}$. Since $\mathbf{S}_{2}$ remains isotropically distributed at $r_{i}$, these subsets are consistent with the initially flat distributions of $\cos \theta_{12}$ and $\Delta \phi$ shown by the horizontal dotted lines. However, the distributions of $\cos \theta_{12}$ and $\Delta \phi$ no longer remain flat as the black holes inspiral to $r_{f}$ as shown by the solid blue and red curves. Those binaries with low (high) values of $\theta_{1}$ at $r_{i}$ have values of $\theta_{12}$ and $\Delta \phi$ at $r_{f}$ strongly peaked about $0^{\circ}\left(180^{\circ}\right)$. This alignment is very pronounced for $q=9 / 11$ both for $\left|\chi_{i}\right|=1$ and $\left|\chi_{i}\right|=0.5$, but it is much less significant for $q=1 / 3$. 

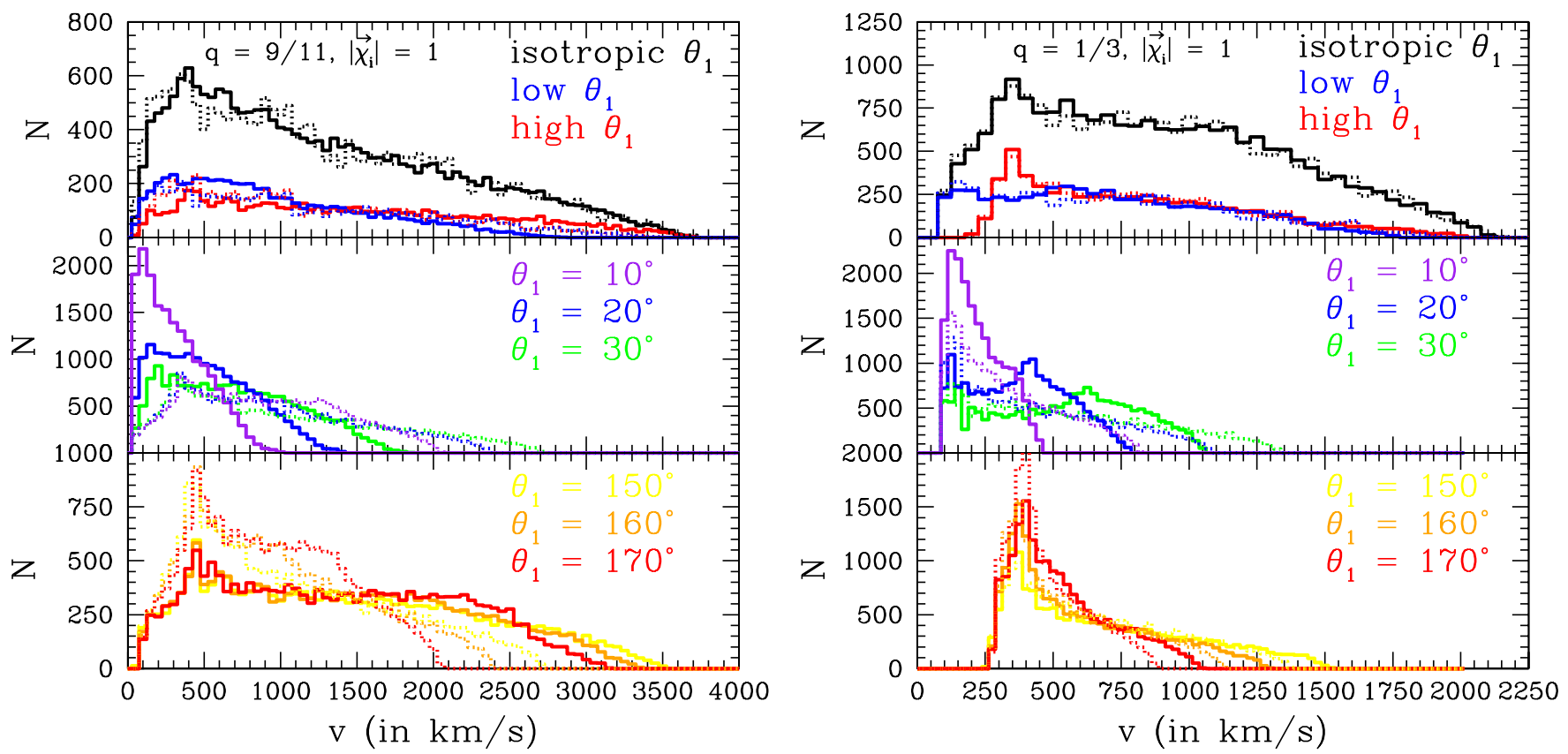

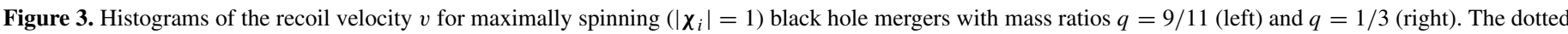

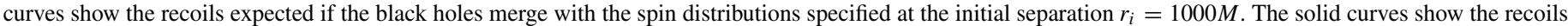

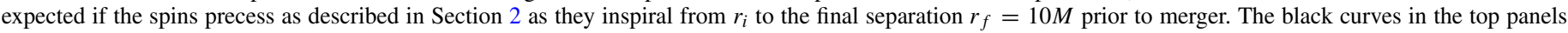

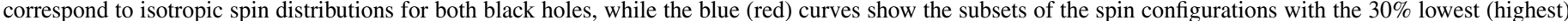

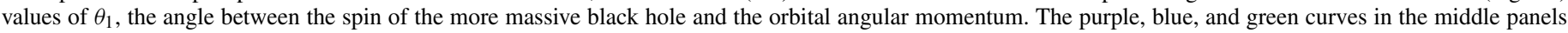

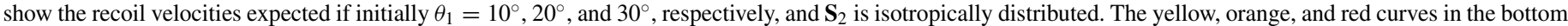
panels show the recoil velocities expected if initially $\theta_{1}=150^{\circ}, 160^{\circ}$, and $170^{\circ}$, respectively, and $\mathbf{S}_{2}$ is isotropically distributed.

(A color version of this figure is available in the online journal.)
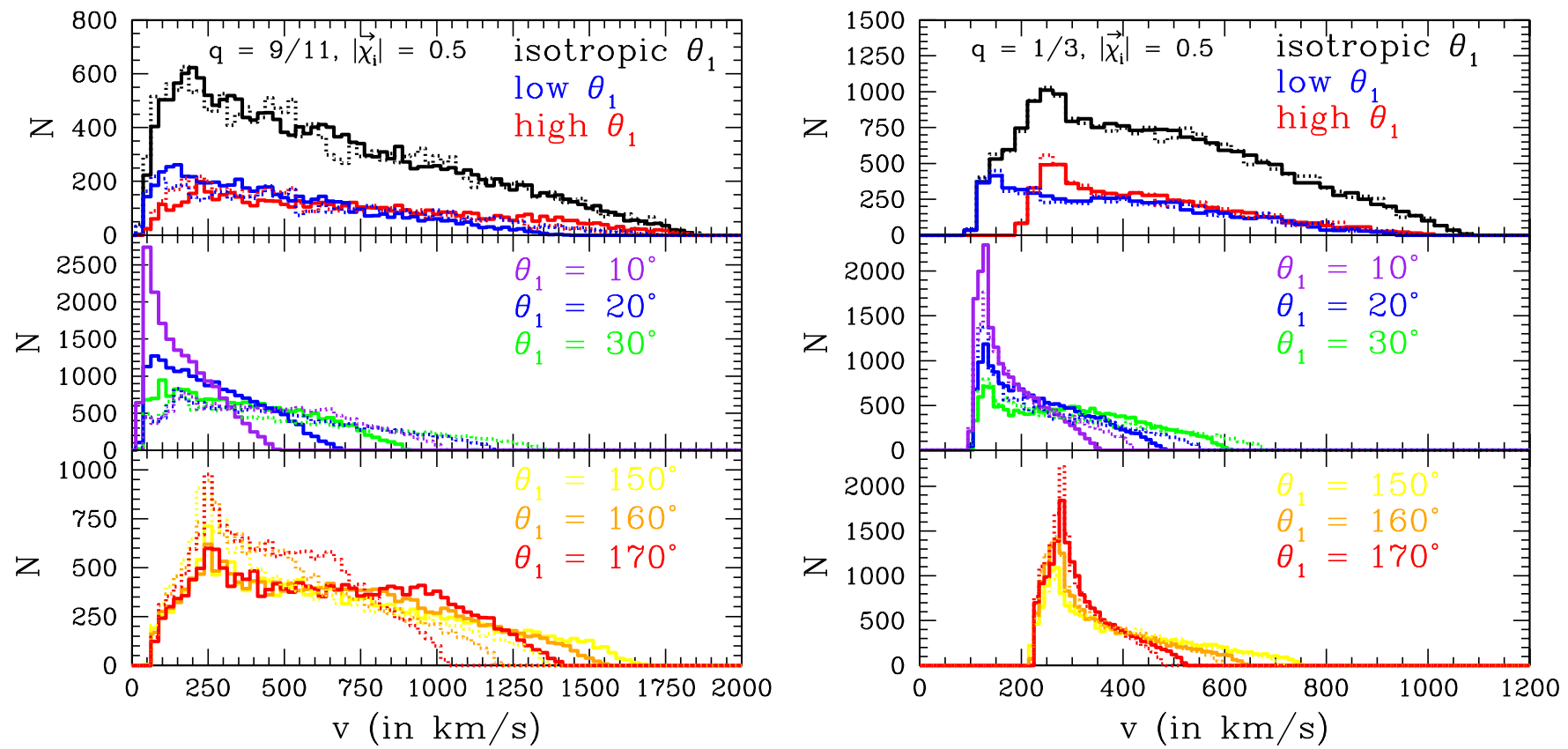

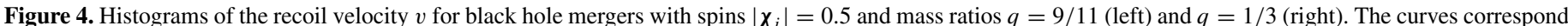
to the same initial spin directions as described in Figure 3.

(A color version of this figure is available in the online journal.)

\section{KICK SUPPRESSION}

How does this alignment of $\mathbf{S}_{1}$ and $\mathbf{S}_{2}$ during the inspiral affect the subsequent recoils? We show histograms of expected recoil distributions for maximally spinning $\left(\left|\chi_{i}\right|=1\right)$ mergers with mass ratios $q=9 / 11$ and $1 / 3$ in Figure 3 , and for mergers with these same mass ratios and spin magnitudes $\left|\chi_{i}\right|=0.5$ in Figure 4. The upper panels of these figures show the recoil distributions for the same binaries whose spin alignment was shown in Figures 1 and 2. The dotted curves in Figures 3 and 4 show the recoils expected if the black holes merged with the same spin distribution they had at $r_{i}$ (no spin precession), while the solid curves show the different recoils expected if we include 
Table 1

Velocities $v_{50}\left(v_{90}\right)$ in $\mathrm{km} \mathrm{s}^{-1}$ at which the Cumulative Distribution Function for Black Hole Recoils Predicted by the RIT Fitting Formula (Campanelli et al. 2007a) Rises Above 50\% (90\%)

\begin{tabular}{|c|c|c|c|c|c|c|c|c|c|c|c|c|c|c|}
\hline \multirow[t]{2}{*}{$\left|\chi_{i}\right|$} & \multirow[t]{2}{*}{$q$} & \multirow[t]{2}{*}{$v$} & \multicolumn{2}{|c|}{$\theta_{1}=10^{\circ}$} & \multicolumn{2}{|c|}{$\theta_{1}=20^{\circ}$} & \multicolumn{2}{|c|}{$\theta_{1}=30^{\circ}$} & \multicolumn{2}{|c|}{$\theta_{1}=150^{\circ}$} & \multicolumn{2}{|c|}{$\theta_{1}=160^{\circ}$} & \multicolumn{2}{|c|}{$\theta_{1}=170^{\circ}$} \\
\hline & & & $1000 M$ & $10 M$ & $1000 M$ & $10 M$ & $1000 M$ & $10 M$ & $1000 M$ & $10 M$ & $1000 M$ & $10 M$ & $1000 M$ & $10 M$ \\
\hline 1.00 & $9 / 11$ & $v_{50}$ & 864 & 273 & 865 & 474 & 889 & 670 & 910 & 1354 & 890 & 1355 & 890 & 1333 \\
\hline 1.00 & $9 / 11$ & $v_{90}$ & 1587 & 611 & 1802 & 947 & 2037 & 1302 & 2047 & 2699 & 1813 & 2567 & 1600 & 2420 \\
\hline 1.00 & $2 / 3$ & $v_{50}$ & 724 & 283 & 724 & 519 & 801 & 707 & 847 & 1031 & 777 & 1035 & 781 & 1060 \\
\hline 1.00 & $2 / 3$ & $v_{90}$ & 1364 & 538 & 1602 & 892 & 1854 & 1252 & 1874 & 2257 & 1627 & 2039 & 1394 & 1930 \\
\hline 1.00 & $1 / 3$ & $v_{50}$ & 290 & 206 & 382 & 384 & 520 & 562 & 601 & 619 & 495 & 521 & 435 & 488 \\
\hline 1.00 & $1 / 3$ & $v_{90}$ & 621 & 364 & 834 & 617 & 1050 & 878 & 1093 & 1183 & 891 & 996 & 697 & 810 \\
\hline 0.75 & $9 / 11$ & $v_{50}$ & 646 & 198 & 647 & 360 & 665 & 516 & 686 & 966 & 671 & 945 & 672 & 997 \\
\hline 0.75 & $9 / 11$ & $v_{90}$ & 1189 & 423 & 1350 & 693 & 1527 & 971 & 1536 & 1942 & 1362 & 1805 & 1203 & 1760 \\
\hline 0.75 & $2 / 3$ & $v_{50}$ & 540 & 210 & 540 & 373 & 599 & 535 & 644 & 785 & 594 & 782 & 597 & 798 \\
\hline 0.75 & $2 / 3$ & $v_{90}$ & 1022 & 439 & 1200 & 711 & 1390 & 1002 & 1410 & 1682 & 1226 & 1525 & 1052 & 1372 \\
\hline 0.75 & $1 / 3$ & $v_{50}$ & 226 & 172 & 294 & 284 & 396 & 411 & 474 & 473 & 400 & 413 & 361 & 388 \\
\hline 0.75 & $1 / 3$ & $v_{90}$ & 469 & 330 & 629 & 513 & 791 & 695 & 832 & 895 & 685 & 747 & 546 & 617 \\
\hline 0.50 & $9 / 11$ & $v_{50}$ & 429 & 142 & 429 & 240 & 442 & 342 & 463 & 609 & 453 & 619 & 453 & 618 \\
\hline 0.50 & $9 / 11$ & $v_{90}$ & 792 & 318 & 899 & 491 & 1018 & 681 & 1027 & 1260 & 910 & 1172 & 805 & 1103 \\
\hline 0.50 & $2 / 3$ & $v_{50}$ & 359 & 183 & 360 & 246 & 400 & 322 & 444 & 506 & 412 & 506 & 416 & 507 \\
\hline 0.50 & $2 / 3$ & $v_{90}$ & 681 & 377 & 800 & 538 & 927 & 679 & 947 & 1083 & 823 & 966 & 711 & 871 \\
\hline 0.50 & $1 / 3$ & $v_{50}$ & 176 & 158 & 217 & 217 & 281 & 294 & 353 & 354 & 310 & 316 & 289 & 301 \\
\hline 0.50 & $1 / 3$ & $v_{90}$ & 326 & 270 & 430 & 374 & 536 & 488 & 577 & 598 & 483 & 509 & 397 & 427 \\
\hline 0.25 & $9 / 11$ & $v_{50}$ & 217 & 123 & 214 & 144 & 221 & 176 & 242 & 287 & 237 & 290 & 238 & 289 \\
\hline 0.25 & $9 / 11$ & $v_{90}$ & 396 & 246 & 450 & 318 & 509 & 398 & 518 & 594 & 461 & 544 & 409 & 503 \\
\hline 0.25 & $2 / 3$ & $v_{50}$ & 189 & 148 & 190 & 161 & 210 & 197 & 251 & 266 & 237 & 261 & 239 & 263 \\
\hline 0.25 & $2 / 3$ & $v_{90}$ & 345 & 274 & 405 & 342 & 468 & 411 & 487 & 524 & 429 & 471 & 375 & 421 \\
\hline 0.25 & $1 / 3$ & $v_{50}$ & 156 & 154 & 169 & 168 & 192 & 193 & 244 & 245 & 231 & 232 & 225 & 227 \\
\hline 0.25 & $1 / 3$ & $v_{90}$ & 207 & 198 & 251 & 241 & 299 & 290 & 334 & 340 & 296 & 301 & 262 & 268 \\
\hline
\end{tabular}

Notes. Entries in each row correspond to distributions with the same spin magnitudes $\left|\chi_{i}\right|$ and mass ratio $q \equiv m_{2} / m_{1}$. The columns indicate the angle $\theta_{1}$ between the spin of the more massive black hole and the orbital angular momentum at $r_{i}=1000 M$, and the binary separation $\left(r_{i}=1000 M\right.$ or $\left.r_{f}=10 M\right)$ at which the spins were used to predict the recoil.

the spin alignment that occurs as the black holes inspiral from $r_{i}$ to $r_{f}$. Comparing the dotted and solid blue curves, we see that spin alignment suppresses the recoils expected if accretion torques at separations $r>r_{i}$ have partially aligned $\mathbf{S}_{1}$ with $\mathbf{L}$ (low $\theta_{1}$ ). The red curves show that recoils are boosted if $\mathbf{S}_{1}$ is initially anti-aligned with $\mathbf{L}$, but we expect this case to be less physically relevant.

Spin alignment and the subsequent suppression of recoils can be even more effective if initially $\theta_{1} \leqslant 30^{\circ}$ as suggested by Dotti et al. (2010). In the middle panels of Figures 3 and 4, we show with purple, blue, and green curves the expected recoils when $\theta_{1}=10^{\circ}, 20^{\circ}$ and $30^{\circ}$ at $r_{i}$ while $\mathbf{S}_{2}$ remains isotropically distributed. The dotted curves show that even with this initial alignment between $\mathbf{S}_{1}$ and $\mathbf{L}$, the distribution has tails that extend to very large recoils. In Table 1, we show the velocities $v_{50}\left(v_{90}\right)$ at which the cumulative distribution function of the recoils rises above $50 \%$ (90\%) for various mass ratios and spins. The first two entries in the first column of this table indicate that even if $\theta_{1}=10^{\circ}$, half the recoils are greater than $864 \mathrm{~km} \mathrm{~s}^{-1}$ and $10 \%$ are greater than $1587 \mathrm{~km} \mathrm{~s}^{-1}$ for $q=9 / 11$ and $\left|\chi_{i}\right|=1$. However, if this spin distribution is allowed to precess as the black holes inspiral from $r_{i}=1000 \mathrm{M}$ to $r_{f}=10 M$, the resulting recoils are dramatically suppressed. The second column shows that for the same mass ratio and spins, $50 \%$ of the recoils are below $273 \mathrm{~km} \mathrm{~s}^{-1}$ and $90 \%$ are below $611 \mathrm{~km} \mathrm{~s}^{-1}$. This reduction is a big effect: most of the black holes produced will now remain bound to normal-sized galaxies. Note that the reduction in recoil velocities remains significant even for relatively moderate spins $\left(\left|\chi_{i}\right| \simeq 0.5\right)$. The precession- induced recoil suppression is less pronounced for lower spins because the spin alignment is reduced and the recoil $v_{m}$ due to the mass asymmetry becomes comparable to the recoils $v_{\perp}, v_{\|}$from the spin asymmetry. In this regime, ejection from the largest galaxies seems unlikely anyway (see, e.g., Figure 2 of Merritt et al. 2004). For completeness, we show how kicks are enhanced for high initial values of $\theta_{1}$ in the bottom panels of Figures 3 and 4 , but do not expect such distributions in astrophysical mergers.

\section{DISCUSSION}

We have shown that as black holes inspiral, spin precession aligns their spins with each other for the spin distributions expected in astrophysical mergers. This spin alignment drastically reduces the recoils expected for the black holes produced in binary mergers. Spin alignment is most effective for the highly spinning, comparable-mass mergers that are predicted to yield the largest recoils (up to $v_{\max }=3750 \mathrm{~km} \mathrm{~s}^{-1}$ according to the fitting formula of Campanelli et al. 2007a). Aligning the black hole spins generically suppresses the recoils (Boyle et al. 2008; Boyle \& Kesden 2008); we found a similar suppression with the alternative fitting formula of Baker et al. (2008). A new recoil formula (van Meter et al. 2010) appeared just before this paper was submitted, which also predicts smaller recoils for aligned spins. This spin alignment is a purely relativistic effect that will occur for all black hole mergers, as GR will always dominate the inspiral for separations $r<r_{\mathrm{GR}} \sim 3000 M$. As long as torques at $r>r_{\mathrm{GR}}$ align $\mathbf{S}_{1}$ and $\mathbf{L}$ such that $\theta_{1} \leqslant 30^{\circ}$, 
spin alignment during the final inspiral will nearly eliminate the $v \gtrsim 1000 \mathrm{~km} \mathrm{~s}^{-1}$ recoils that are so difficult to reconcile with galaxies keeping their SBHs. While there is still great uncertainty about how merging black holes interact with surrounding gas, the PN spin precession discussed in this paper is inevitable and results from well-established physics. We therefore believe that spin alignment must be accounted for in future population studies of merging black holes.

We are grateful to Vitor Cardoso for testing our PN evolution code. We also thank Enrico Barausse, Manuela Campanelli, Yanbei Chen, Pablo Laguna, Carlos Lousto, Samaya Nissanke, Evan Ochsner, Sterl Phinney, Étienne Racine, Luciano Rezzolla, and Manuel Tiglio for useful discussions. This work was supported by grants from the Sherman Fairchild Foundation to Caltech, by NSF grant Nos. PHY-0601459 (PI: Thorne) and PHY-090003 (TeraGrid), and by FCT-Portugal through projects PTDC/CTE-AST/098034/2008 and PTDC/ FIS/098032/2008. M.K. acknowledges support from the NASA BEFS grant NNX07AH06G (PI: Phinney). E.B.'s and U.S.'s research was supported by NSF grants PHY-0900735 and PHY0652995, respectively.

\section{REFERENCES}

Baker, J. G., Boggs, W. D., Centrella, J., Kelly, B. J., McWilliams, S. T., Miller, M. C., \& van Meter, J. R. 2008, ApJ, 682, L29

Baker, J. G., Centrella, J., Choi, D. I., Koppitz, M., \& van Meter, J. 2005, Phys. Rev. Lett., 96, 111102

Bardeen, J. M. 1970, Nature, 226, 64

Begelman, M. C., Blandford, R. D., \& Rees, M. J. 1980, Nature, 287, 307

Berti, E., Cardoso, V., \& Starinets, A. O. 2009, Class. Quantum Grav., 26, 163001

Berti, E., \& Volonteri, M. 2008, ApJ, 684, 822

Bogdanović, T., Reynolds, C. S., \& Miller, M. C. 2007, ApJ, 661, L147

Boyle, L., \& Kesden, M. 2008, Phys. Rev. D, 78, 024017
Boyle, L., Kesden, M., \& Nissanke, S. 2008, Phys. Rev. Lett., 100, 151101

Brenneman, L. W., \& Reynolds, C. S. 2006, ApJ, 652, 1028

Campanelli, M., Lousto, C. O., Marronetti, P., \& Zlochower, Y. 2006, Phys. Rev. Lett., 96, 111101

Campanelli, M., Lousto, C. O., Zlochower, Y., \& Merritt, D. 2007a, ApJ, 659, L5

Campanelli, M., Lousto, C. O., Zlochower, Y., \& Merritt, D. 2007b, Phys. Rev. Lett., 98, 231102

Chang, P., Strubbe, L. E., Menou, K., \& Quataert, E. 2009, MNRAS, submitted (arXiv:0906.0825)

Dotti, M., Volonteri, M., Perego, A., Colpi, M., Ruszkowski, M., \& Haardt, F. 2010, MNRAS, 402, 682

Ferrarese, L., \& Merritt, D. 2000, ApJ, 539, L9

Gonzalez, J. A., Hannam, M. D., Sperhake, U., Bruegmann, B., \& Husa, S. 2007a, Phys. Rev. Lett., 98, 231101

Gonzalez, J. A., Sperhake, U., Bruegmann, B., Hannam, M., \& Husa, S. 2007b, Phys. Rev. Lett., 98, 091101

Herrmann, F., Silberholz, J., Bellone, M., Guerberoff, G., \& Tiglio, M. 2010, Class. Quantum Grav., 27, 032001

Kesden, M., Sperhake, U., \& Berti, E. 2010, Phys. Rev. D, in press (arXiv: 1002.2643)

Kidder, L. E. 1995, Phys. Rev. D, 52, 821

King, A. R., \& Pringle, J. E. 2007, MNRAS, 377, L25

Lousto, C. O., Nakano, H., Zlochower, Y., \& Campanelli, M. 2009, arXiv:0910.3197

Lousto, C. O., \& Zlochower, Y. 2008, Phys. Rev. D, 77, 044028

Lousto, C. O., \& Zlochower, Y. 2009, Phys. Rev. D, 79, 064018

Magorrian, J., et al. 1998, AJ, 115, 2285

Merritt, D., Milosavljevic, M., Favata, M., Hughes, S. A., \& Holz, D. E. 2004, ApJ, 607, L9

Peters, P. C., \& Mathews, J. 1963, Phys. Rev., 131, 435

Pretorius, F. 2005, Phys. Rev. Lett., 95, 121101

Racine, E. 2008, Phys. Rev. D, 78, 044021

Reynolds, C. S., Young, A. J., Begelman, M. C., \& Fabian, A. C. 1999, ApJ, 514,164

Schnittman, J. D. 2004, Phys. Rev. D, 70, 124020

Schnittman, J. D. 2007, ApJ, 667, L133

Schnittman, J. D., \& Buonanno, A. 2007, ApJ, 662, L63

Tremaine, S., et al. 2002, ApJ, 574, 740

van Meter, J. R., Miller, M. C., Baker, J. G., Boggs, W. D., \& Kelly, B. J. 2010 arXiv: 1003.3865 